\title{
Varón de 74 años que ingresa por insuficiencia cardíaca: ¿sólo insuficiencia cardíaca?
}

\author{
Francisco López-García', Cristina Martínez-Solís', María del Pilar Benito-Muñoz ${ }^{2}$ \\ ${ }^{1}$ Servicio de Medicina Interna. Hospital General Universitario de Elche. Elche (Alicante). España \\ ${ }^{2}$ Estudiante de Medicina. Universidad Miguel Hernández. Elche (Alicante). España
}

Recibido: 03/04/2019

Aceptado: 03/04/2019

En línea: 30/04/2019

Citar como: López-García F, Martínez-Solís C, Benito-Muñoz MP. Varón de 74 años que ingresa por insuficiencia cardíaca: ¿sólo insuficiencia cardíaca? Rev Esp Casos Clin Med Intern (RECCMI). 2019 (Abr); 4(1): 39-41. doi: 10.32818/reccmi.a4n1a14.

Autor para correspondencia: Francisco López-García.filoflg1968@gmail.com

\begin{tabular}{l} 
Palabras clave \\
\hline$\triangleright$ Enfermedad pulmonar \\
obstructiva crónica \\
$\triangleright$ Insuficiencia \\
cardíaca crónica \\
$\triangleright$ Comorbilidades
\end{tabular}

Keywords

$\triangleright$ Chronic obstructive pulmonary disease

$\triangleright$ Heart chronic failure

$\triangleright$ Comorbidities

\begin{abstract}
Resumen
Varón de 74 años, fumador, con antecedentes de hipertensión arterial, diabetes mellitus tipo 2, dislipemia, fibrilación auricular permanente e insuficiencia cardíaca crónica que ingresó por descompensación cardíaca aguda. La respuesta al tratamiento con diuréticos fue muy buena y el paciente fue dado de alta con solicitud de ecocardiografía y espirometría en fase de estabilidad clínica de su insuficiencia cardíaca. La espirometría fue diagnóstica de una enfermedad pulmonar obstructiva crónica (EPOC) grave, por lo que fue tratado con broncodilatadores. El caso ilustra la problemática del infradiagnóstico de la EPOC en pacientes con insuficiencia cardíaca crónica, a pesar de que ambas enfermedades coexisten con mucha frecuencia en el mismo paciente.

Abstract
A 74-year-old male smoker with a history of hypertension, DM type 2, dyslipidemia, permanent AF, and chronic
heart failure (HF) who was admitted due to acute cardiac decompensation. The response to treatment with diu-
retics was very good and the patient was discharged with a request for echocardiography and spirometry in the
phase of clinical stability of his HF. Spirometry was diagnostic of a severe COPD, which was undiagnosed, so it was
treated with bronchodilators. The case illustrates the problem of the underdiagnosis of COPD in patients with HF
even though both diseases coexist very frequently in the same patient.
\end{abstract}

\section{Puntos destacados}

$\triangleright$ La insuficiencia cardíaca crónica (IC) y la enfermedad pulmonar obstructiva crónica (EPOC) coexisten en un mismo paciente en un elevado porcentaje de casos. Hasta el $25 \%$ de los pacientes con IC tienen EPOC y alrededor del 30\% de los pacientes con EPOC tienen IC.

$\triangleright$ Es necesario evitar el infradiagnóstico de ambas enfermedades cuando se presentan juntas en un paciente. Esto implica realizar espirometría en los pacientes fumadores con IC, y ecocardiografía en pacientes fumadores con EPOC, sobre todo si tienen síntomas.

$\triangleright$ Debe planificarse el tratamiento de estas dos enfermedades de forma conjunta, con el objetivo de disminuir la morbimortalidad de ambas.

\section{Introducción}

En el marco de las enfermedades cardiovasculares, la insuficiencia cardíaca crónica (IC) es la enfermedad más prevalente y genera una importante morbi- mortalidad y consumo de recursos sanitarios. Por otra parte, dentro de las enfermedades respiratorias, la enfermedad pulmonar obstructiva crónica (EPOC) es la patología más frecuente y también se asocia con discapacidad, deterioro de la calidad de vida y aumento de la mortalidad en el paciente que la padece ${ }^{1,2}$.

En la práctica clínica, estas dos entidades se asocian en un mismo paciente, dado que comparten mecanismos etiopatogénicos (tabaco, inflamación sistémica) y también debido a su alta frecuencia, sobre todo en pacientes crónicos y de edad avanzada. De hecho, ambas enfermedades se identifican con el prototipo de enfermedad crónica, están presentes en pacientes pluripatológicos y se asocian claramente al envejecimiento ${ }^{3}$. A pesar de su frecuente asociación, y a las claras implicaciones diagnósticas y terapéuticas que implica el reconocimiento de ambas entidades en el mismo paciente, es muy común que la IC no se tenga en cuenta en el paciente con EPOC; y viceversa, que no se diagnostique la EPOC en pacientes con IC. 
El caso clínico presentado es el de un paciente con IC con historia de disnea de larga evolución al que se diagnosticó de EPOC grave después de realizársele una espirometría posbroncodilatación.

\section{Caso clínico}

Varón de 74 años que ingresó en Medicina Interna por disnea. Sus antecedentes eran hipertensión arterial, dislipemia, diabetes mellitus tipo 2, fibrilación auricular permanente e IC con FEVI preservada. Grado 2 de disnea según la escala NYHA, sin edemas crónicos en miembros inferiores. Entre sus hábitos tóxicos reconocía tabaquismo activo de 50 paquetes/año. Recibía tratamiento con metformina 850 mg/12 h, vildagliptina 50 mg/12 h, valsartán 160 mg/24 h, hidroclorotiazida 25 mg/24 h, bisoprolol 5 mg/24 h, atorvastatina 40 mg/24 h y apixaban $5 \mathrm{mg} / 12 \mathrm{~h}$

Ingresó en el Servicio de Medicina Interna por presentar, desde 3 días antes del ingreso, aumento de su disnea, que se hizo de mínimo esfuerzo con ortopnea de dos almohadas y aparición de crisis de disnea paroxística nocturna. Le habían aumentado claramente los edemas en las piernas. No refería fiebre, distermia, dolor torácico o abdominal, ni aumento de la tos, producción de esputo o hemoptisis. En el área de Urgencias se constató un paciente consciente y orientado, con regular estado general, afebril, taquipneico y que presentaba una saturación arterial basal de oxígeno del 90\%. Tensión arterial 130/70 mmHg. No presentaba ictericia, cianosis o acropaquias. La auscultación cardíaca mostró una frecuencia arrítmica a 88 lpm sin soplos ni roces, y en la auscultación pulmonar existían crepitantes teleinspiratorios bilaterales, sobre todo en hemitórax derecho. La exploración abdominal y la neurológica fueron normales. En ambos miembros inferiores eran evidentes edemas con fóvea sin signos de trombosis venosa profunda.

El hemograma mostró hemoglobina 12,7 g/dl, leucocitos 8.660 (75\% neutrófilos), plaquetas 328.000. La bioquímica con función hepática, renal, iones y triglicéridos fueron normales. El valor de la proteína $C$ reactiva $(P C R)$ fue de 6 mg/l y el del NT-proBNP de 9.600 pg/ml. El electrocardiograma mostró un ritmo de fibrilación auricular a 88 lpm de respuesta ventricular media, sin signos agudos de isquemia ni alteraciones de la repolarización. La radiografía de tórax mostró cardiomegalia y derrame pleural bilateral con signos de insuficiencia ventricular izquierda (Figura 1).

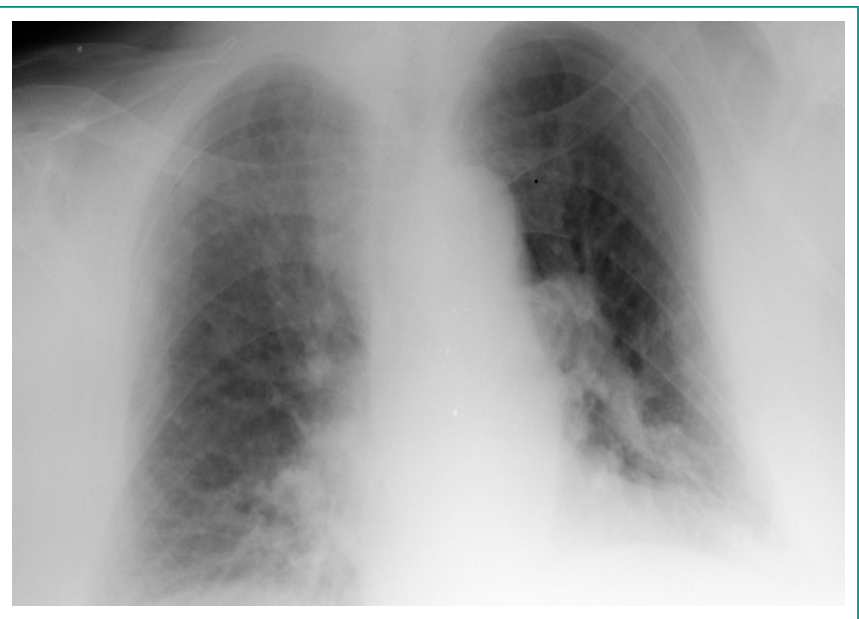

Figura 1. Radiografía de tórax al ingreso

Con el diagnóstico de insuficiencia cardíaca, el paciente fue tratado con oxigenoterapia, restricción de líquidos y furosemida intravenosa. Su evolución clínica fue muy favorable, mejorando su disnea hasta la suya en situación basal, así como la congestión pulmonar y los edemas en las piernas. Se realizó una radiografía de tórax (Figura 2), que mostró desaparición de los signos de insuficiencia cardíaca.

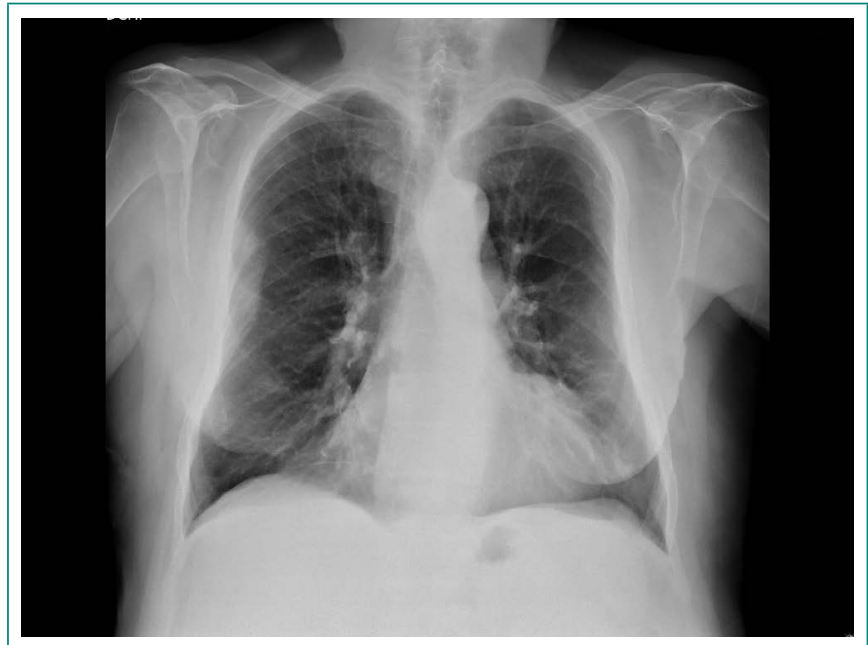

Figura 2. Radiografía de tórax al alta

El paciente fue dado de alta con aumento en el tratamiento diurético y se citó en las consultas externas de Medicina Interna al mes del alta hospitalaria para realizar una ecocardiografía y una espirometría con test posbroncodilatador. En la valoración en la consulta externa de Medicina Interna, el paciente refería mejoría de la disnea, aunque persistía en situación de esfuerzos moderados. No tenía edemas y la auscultación cardíaca y la pulmonar fueron normales. La ecocardiografía mostró un ventrículo izquierdo no dilatado, con hipertrofia concéntrica moderada, FEVI global y contractilidad segmentaria normal. Disfunción diastólica grado II con aumento de las presiones de llenado de la aurícula izquierda, que estaba moderadamente dilatada. No valvulopatías significativas. Ventrículo derecho no dilatado. Insuficiencia tricuspídea ligera con baja probabilidad de hipertensión pulmonar. No derrame pericárdico. Por su parte, la espirometría posbroncodilatación mostró FEV1/FVC 52\%, FEV1 830 (37\%), FVC 1.620 (54\%). La prueba broncodilatadora fue negativa.

Con el diagnóstico de EPOC con afectación espirométrica grave, se inició tratamiento con indacaterol + glicopirronio en dispositivo Breezhaler ${ }^{\circledR}$ en dosis única diaria y se mantuvo el tratamiento de su insuficiencia cardíaca con reducción en la dosis de diurético.

\section{Discusión}

Un aspecto relevante de la IC y de la EPOC es que ambas se asocian con múltiples comorbilidades. En la IC, las comorbilidades más frecuentes son trastornos respiratorios del sueño, insuficiencia renal, anemia, diabetes mellitus, trastornos musculoesqueléticos, deterioro cognitivo y depresión ${ }^{4}$. En los pacientes con IC, la prevalencia de EPOC oscila entre el 10-40\%, dependiendo de las series y del entorno clínico estudiado. El estudio GESAIC mostró una prevalencia de EPOC del 25\% en 391 pacientes hospitalizados en 15 servicios de Medicina Interna ${ }^{5}$. Por otra parte, en los pacientes con EPOC, la presencia de comorbilidades es la norma, siendo las más frecuentes hipertensión arterial, diabetes mellitus, dislipemia, enfermedad coronaria, anemia, insuficiencia renal crónica, ansiedad y depresión. La presencia de IC en los pacientes con EPOC es muy alta; en los estudios ECCO y ESMI, realizados por el Grupo de EPOC de la Sociedad Española de Medicina Interna (SEMI) y que evaluaron 
las comorbilidades presentes en pacientes ingresados por agudización de EPOC, la prevalencia de IC fue del 27-32\% ${ }^{6}$.

El diagnóstico de la EPOC en pacientes con IC no es fácil, debido a múltiples causas. Entre ellas, cabe destacar que los síntomas (sobre todo la disnea) son comunes a ambas enfermedades, que los péptidos natriuréticos pierden capacidad de precisión diagnóstica, que los patrones radiológicos son confusos, que las pruebas espirométricas sobreestiman la EPOC en el paciente con IC, y que hasta el $40 \%$ de resultados en la ecocardiografía no llegan a ser concluyentes ${ }^{7}$. A pesar de todo, es muy importante investigar de forma sistemática en todos los pacientes fumadores con IC la presencia de EPOC no diagnosticada realizando una espirometría en fase de estabilidad clínica de la IC (ausencia de congestión pulmonar). Por otra parte, en los pacientes con IC y EPOC deben seguirse las recomendaciones de las guías de práctica clínica sobre las enfermedades individuales ${ }^{8}$. De hecho, múltiples estudios han demostrado que el tratamiento con betabloqueantes cardioselectivos es seguro en los pacientes con IC que tienen EPOC, incluso disminuye los ingresos hospitalarios y mues tra beneficios en la mortalidad ${ }^{9}$. Por otra parte, ensayos clínicos aleatorizados y controlados realizados en pacientes con EPOC no han demostrado que el tratamiento con betaagonistas de acción larga y/o anticolinérgicos de acción prolongada empeoren la mortalidad cardiovascular o la incidencia de $\mathrm{IC}^{10}$ El caso presentado es representativo de un paciente con IC, fumador, con disnea de larga evolución que tenía EPOC infradiagnosticada con afectación espirométrica grave. La disnea es el síntoma más frecuente de ambas enfermedades y en su aparición y persistencia influyen tanto la IC como la EPOC, por lo que es necesario el diagnóstico precoz de las dos patologías. Es razonable concluir que en las guías de práctica clínica cardiológicas de la IC se debería incluir la espirometría posbroncodilatación como prueba de cribado obligatoria en todo paciente con IC y síntomas respiratorios, sobre todo si es fumador. La optimización del tratamiento de ambas enfermedades podría mejorar la calidad de vida y el pronóstico de los pacientes que las padecen.

\section{Bibliografía}

1. Sidney S, Sorel M, Quesenberry Jr CP, et al. COPD and incident cardiovascular disease hospitalizations and mortality: Kaiser Permanente Medical Care Program. Chest. 2005; 128: 2068-2075. doi: 10.1378/chest.128.4.2068.
2. Global for the Diagnosis, Management and Prevention of COPD, Global initiative for Chronic Obstructive Lung Disease (GOLD) 2019. Disponible en: http://goldcopd.org.

3. Manzano L, Yebra-Yebra M. Insuficiencia cardíaca y enfermedad pulmonar obstructiva crónica: un enfermo con dos enfermedades. Med Clin (Barc). 2010; 134(10): 443-444. doi: 10.1016/j.medcli.2009.11.023.

4. Montero Pérez-Barquero M, Conthe Gutiérrez P, Román Sánchez P, García Alegría J, Forteza-Rey J; Grupo de Trabajo de Insuficiencia Cardíaca de la Sociedad Española de Medicina Interna (estudio SEMI-IC). [Morbidity from patients admitted for heart failure in the departments of internal medicine]. Rev Clin Esp. 2010 Apr; 210(4): 149-158. doi: 10.1016/j. rce.2009.09.007.

5. Recio-Iglesias J, Grau-Amorós J, Formiga F, et al, en nombre de los investigadores del Grupo para el Estudio y Significado de la Anemia en la Insuficiencia Cardíaca (GESAIC). Enfermedad pulmonar obstructiva crónica en pacientes ingresados por insuficiencia cardíaca. Resultados del Grupo para el Estudio y Significado de la Anemia en la Insuficiencia Cardiaca (GESAIC). Med Clin (Barc). 2010; 134: 427-432. doi: 10.1016/j.medcli.2009.09.046.

6. Almagro P, López F, Cabrera FJ, Portillo J, Fernández-Ruiz M, Zubillaga E, et al; Grupos de Trabajo de EPOC y Paciente Pluripatológico y Edad Avanzada de la Sociedad Española de Medicina Interna. Comorbilidades en pacientes hospitalizados por enfermedad pulmonar obstructiva crónica. Análisis comparativo de los estudios ECCO y ESMI. Rev Clin Esp. 2012 Jun; 212(6): 281-286. doi: 10.1016/j.rce.2012.02.014

7. Hawkins NM, Petrie MC, Jhund PS, Chalmers GW, Dunn FG, McMurray J. Heart failure and chronic obstructive pulmonary disease: diagnostic pitfalls and epidemiology. Eur J Heart Fail. 2009 Feb; 11(2): 130-139. doi: 10.1093/ eurjhf/hfn013.

8. Recio Iglesias J. Insuficiencia cardíaca. En: Protocolos: manejo diagnóstico y terapéutico de las comorbilidades en la EPOC. 1. a ed. Díez Manglano J, López García F. Madrid. Elsevier Doyma Editores, 2014; 71-81.

9. Etminan M, Jafari S, Carleton B, FitzGerald JM. Beta-blocker use and COPD mortality: a systematic review and meta-analysis. BMC Pulm Med. 2012 Sep 4; 12: 48. doi: 10.1186/1471-2466-12-48.

10. Au DH, Udris EM, Curtis JR, McDonell MB, Fihn SD; ACQUIP Investigators. Association between chronic heart failure and inhaled beta-2-adrenoceptors agonists. Am Heart J. 2004; 148: 885-891. doi: 10.1016/j.ahj.2004.03.048 